\title{
Human Muscle Cultured in Monolayer and Cocultured with Fetal Rat Spinal Cord: Importance of Dorsal Root Ganglia for Achieving Successful Functional Innervation
}

\author{
Takayoshi Kobayashi, ${ }^{a}$ Valerie Askanas, and W. King Engel \\ USC Neuromuscular Center, Department of Neurology, University of Southern California School of Medicine, Los Angeles, \\ California 90017
}

Adult human muscle cultured in monolayer was cocultured with explants of 13-14-d-old rat embryo using (a) ventral spinal cord (VSC), (b) transverse section of whole spinal cord (WSC), and (c) WSC with dorsal root ganglia (DRG) attached (WSC + DRG). AChR clusters and AChE-positive patches, both at the nerve-muscle contacts, were studied at 5, 12, and $21 \mathrm{~d}$ of coculture with each of the 3 spinal cord preparations. In addition, AChE-positive patches were studied after 31-64 d of coculture with WSC + DRG to evaluate further organization of those patches.

Compared to VSC and WSC cocultures, WSC + DRG induced significantly more AChR clusters per muscle fiber at the nerve-muscle contacts at $5 \mathrm{~d}$ of coculture, and the percentage of muscle fibers containing AChR clusters was higher at all 3 time points quantitated. The number of AChE-positive sites was the same with all 3 spinal cord preparations in early (day 5) cocultures. Between 12 and $21 \mathrm{~d}$ of coculture, the number of muscle fibers containing AChE patches increased significantly only with WSC + DRG, correlating with the increased number of contracting muscle fibers in that coculture system.

Only in human muscle cocultured with WSC + DRG was successful innervation of the cultured muscle fibers achieved, as manifested by (1) contractions in a continuous rhythm of large groups of muscle fibers that were reversibly blocked by $1 \mathrm{~mm} d$-tubocurarine (aneurally cultured human muscle does not spontaneously contract); (2) well-developed crossstriations throughout the fiber; (3) well-organized AChE-positive sites; and (4) a trend from multifocal toward unifocal innervation of those muscle fibers.

Our studies demonstrate that adult human muscle cultured in monolayer can be innervated by fetal rat spinal cord and that, in our system, DRG are essential for achieving functional innervation.

De novo formation of neuromuscular junctions in monolayer cultures has been demonstrated previously in embryonic animal muscle and animal muscle cell lines cocultured with embryonic

Received Sept. 23, 1986; revised Mar. 25, 1987; accepted Apr. 1, 1987.

This work was supported by U.S. Public Health Service Grant NS 18661 to V.A. T.K. was a Muscular Dystrophy Association Postdoctoral Research Fellow. Correspondence should be addressed to Valerie Askanas, USC Neuromuscular Center, IIospital of the Good Samaritan, 637 South Lucas Avenue, Los Angeles, CA 90017-2395.

a Present address: Department of Neurology, Tokyo Medical and Dental University, 1-4-45 Ushima, Bunkyo-ku, Tokyo 113, Japan.

Copyright (C 1987 Society for Neuroscience $0270-6474 / 87 / 103131-11 \$ 02.00 / 0$ animal spinal cord (Shimada et al., 1969; Fischbach, 1972; Fischbach et al., 1979; Nakajima et al., 1980; Davey and Cohen, 1986). Recently, we have developed a new model in which adult human muscle cultured in monolayer can be cocultured with fetal rat spinal cord. In this system, nicotinic AChR and AChE accumulate at the nerve-muscle contacts, presumably young neuromuscular junctions that have developed entirely de novo in culture (Kobayashi and Askanas, 1985). Only when the dorsal root ganglia (DRG) were attached to the spinal cord explant did large areas of cross-striated human muscle fibers in close proximity to the ventral part of spinal cord cxplant contract in a continuous rhythm, and those contractions could be reversibly blocked by $1 \mathrm{~mm}$ of $d$-tubocurarine (Askanas et al., 1985; Kobayashi et al., 1985). Coculture with whole transverse explants of the spinal cord without DRG attached, or with the explants of the ventral part of spinal cord (VSC), caused accumulation of AChR clusters at the nerve-muscle contacts and survival of the cultured human muscle longer than when cultured aneurally; but only occasionally did those 2 types of spinal cord coculture induce contractile activity of the muscle fibers. Unlike aneurally cultured embryonic animal muscle, which practically always spontaneously contracts, human muscle fibers cultured aneurally in monolayer virtually never spontaneously contract (Askanas and Engel, 1979).

Our previous report (Kobayashi and Askanas, 1985) described only the phenomena of AChR and AChE accumulation at the nerve-muscle contacts. In the present study we describe quantitative analyses of $\mathrm{AChR}$ clusters and AChE-positive patches formed at nerve-muscle contacts at various stages of innervation of human muscle cultured in monolayer and then cocultured with 3 different kinds of spinal cord explants, ventral spinal cord (VSC), whole spinal cord (WSC), and WSC with DRG attached (WSC + DRG). Spatial and quantitative relationships between $\mathrm{AChR}$ clusters and $\mathrm{AChE}$ patches were also studied at various developmental stages of neuromuscular junction formation, applying a technique we developed of doublelabeling $\mathrm{AChR}$ and $\mathrm{AChE}$ on the basis of autoradiography of ${ }^{125}$ alpha-bungarotoxin and histochemistry of $\mathrm{AChE}$. The lengths and widths of innervated-contracting and innervated-noncontracting control muscle fibers were also measured.

\section{Materials and Methods}

Coculture of human muscle grown in monolayer with fetal rat spinal cord. Human muscle cultures were established, using our explant-reexplantation technique (Askanas and Engel, 1975), from diagnostic muscle biopsies of patients considered, after all studies were performed, not to have an intrinsic muscle disease. Cultures were initiated from $1 \mathrm{~mm}^{3}$ 
muscle explants, 5 of which were placed in each $35 \mathrm{~mm}$ petri dish (Falcon) coated with a gelatin-human plasma mixture. After an abundant growth of cells had emerged from the explants, the explants were removed and transferred to collagen-coated petri dishes. After another abundant growth of cells had emerged from the reexplanted explants, those explants were removed and the monolayer muscle cultures continued. About 5-7 d after myoblast fusion, $1 \mathrm{~mm}^{3}$ explants of spinal cord derived from 13-14-d-old Sprague-Dawley rat embryos $(8-10 \mathrm{~mm}$ in length) were placed on the myotube monolayer cultures, 5 explants per petri dish.

Seventeen different experiments were established, comprising a total of 680 cultures, with 3 different types of spinal cord, viz., VSC, WCS, and WCS + DRG. An additional 23 experiments, comprising a total of 660 cultures, served for coculture of human muscle and WSC + DRG for studies of contracting muscle.

Before coculture with spinal cord, the muscle alone was cultured in $F_{14}$ medium (Gibco) (Vogel et al., 1972) supplemented with $10 \%$ fetal bovine serum (FBS), $50 \mathrm{ng}$ fibroblast growth factor (FGF), $10 \mathrm{ng}$ epidermal growth factor (EGF), and $10 \mu \mathrm{g}$ insulin $/ 1 \mathrm{ml}$ medium (Askanas and Gallez-Hawkins, 1985). After coculture with spinal cord explants, cultures were maintaincd in $\mathrm{F}_{14}$ medium supplementcd with $10 \%$ FBS. All cultures were fed twice a week, and living cultures were examined every or every other day by phase-contrast inverted microscopy.

Quantitative analysis of AChR clusters on human muscle fibers cocultured with different types of spinal cord preparations. Seven independent experiments were established to study the presence of AChR clusters at nerve-muscle contacts of human muscle cocultured with VSC, WSC, and WSC + DRG for 5, 12, and $21 \mathrm{~d}$. Six to twelve culture dishes were established for each type of spinal cord explant. In each experiment, AChR clusters were visualized by autoradiography of ${ }^{125}$ I-alpha-bungarotoxin (alpha-BT). Autoradiography of alpha-BT was performed as described previously (Kobayashi and Askanas, 1985). Large areas of muscle fibers contacted by neurites were chosen for quantitative analysis of AChR clusters. AChR clusters were counted in 2-4 fields per culture dish using a $6.3 \times$ bright-field objective. The total number of muscle fibers that appeared to be contacted by neurites and the total number of AChR clusters were counted. Accordingly, the number of AChR clusters per individual muscle fiber was calculated. Since a few of the muscle fibers that appeared, under the light microscopy, to be contacted by neurites may have only had neurites trespassing them, the total number of muscle fibers counted in each type of coculture was large, in an attempt to compensate for this unavoidable error. The number of noncontracting muscle fibers counted in each type of coculture at 5,12 , and $21 \mathrm{~d}$ was 923,1077 , and 676 , respectively, in VSC; 619,1267 , and 604 for WSC; and 1609, 1041, and 884 for WSC + DRG. The same calculations were performed on 271 continuously contracting muscle fibers at $21 \mathrm{~d}$ of WSC + DRG coculture.

Quantative analysis of AChE patches on human muscle fibers cocultured with different types of spinal cord explants. AChE staining was performed as previously described (Kobayashi and Askanas, 1985), according to a modification of the method of Karnowsky and Roots (1964) using rubeanic acid for enhancement (Nakamura and Torigoe, 1979; Kobayashi et al., 1982). Five independent experiments with human muscle cocultured with 3 different kinds of spinal cord explants were studied at 5,12 , and $21 \mathrm{~d}$ of coculture. The number of muscle fibers counted at those 3 time points was 587,541 , and 443 , respectively, for VSC; 640,463 , and 515 for WSC; and 577,508 , and 538 for WSC + DRG. In addition, AChE-positive patches on 201 contracting muscle fibers and on 163 noncontracting but neurite-contacted muscle fibers were counted in 7 culture dishes from 5 experiments at 31-64 d after coculture, since well-organized AChE-positive patches were easily identifiable in the older cocultures. Quantitative analysis of the AChE patches was performed in the same way as analysis of AChR clusters.

Double-labeling of AChR clusters and AChE patches by combining autoradiography of ${ }^{125} I$-alpha-BT and AChE histochemistry. To study the relationship between AChR clusters and AChE patches on the same muscle fiber, cultures were "stained" with a double-labeling method we developed. Living cultures were first incubated at $37^{\circ} \mathrm{C}$ with ${ }^{125} \mathrm{I}$-alphaBT for $1 \mathrm{hr}$. After incubation, cultures were rinsed several times in $F_{14}$ medium with $0.2 \% \mathrm{BSA}$ and $0.1 \mathrm{M}$ phosphate buffer, fixed for $5 \mathrm{~min}$ in $1 \%$ glutaraldehyde in $0.1 \mathrm{M}$ phosphate buffer, and rinsed 3 times in 0.1 M phosphate buffer. After fixation, cultures were incubated at $37^{\circ} \mathrm{C}$ for $60 \mathrm{~min}$ in a histochemical medium for AChE containing $5 \mathrm{mg}$ acetylthiocholine iodide, $6.3 \mathrm{ml}$ of $0.2 \mathrm{M}$ phosphate buffer (pH 5.8$), 0.7 \mathrm{ml}$ of $0.1 \mathrm{M}$ sodium citrate, $1.0 \mathrm{ml}$ of $30 \mathrm{~mm}$ copper sulfate, $1.0 \mathrm{ml}$ of 0.2
mM iso-octamethyl pyrophosphoramide (OMPA), and $1.0 \mathrm{ml}$ of $5 \mathrm{~mm}$ potassium ferricyanide. After incubation, cultures were thoroughly rinsed, refixed again with $2.5 \%$ glutaraldehyde $+1 \%$ tannic acic for $30 \mathrm{~min}$, and rinsed several times with $0.1 \mathrm{M}$ phosphate buffer. AChE-positive patches were then photographed in bright- and phase-contrast microscopy. After being photographed, the cultures were dehydrated, covered with NTB II cmulsion, and exposed for $9 \mathrm{~d}$ in the dark at $4^{\circ} \mathrm{C}$. After the autoradiographs were developed, the exact areas of those autoradiographs were matched with the previously taken AChE pictures, and the autoradiographs were photographed at the same magnification as were the AChE stains.

The double-labeling method was performed after 13-41 d of coculture with WSC + DRG on cultures containing both large areas of contracting muscle fibers and, for comparison, areas of muscle fibers contacted by neurites but not contracting.

The total number of AChR clusters and AChE patches, and the length of AChR clusters and AChE patches, were measured on the same individual muscle fibers. The relationship between AChR clusters and AChE patches was studied on 632 contracting and 247 noncontracting muscle fibers.

Quantitative analysis of the length and the width of contracting and noncontracting muscle fibers contacted by rat spinal cord neurons. Contracting muscle fibers were present only in cocultures with WSC + DRG. Irregular contractions were visible after 7-14 $\mathrm{d}$ of muscle-spinal cord coculture. About 2 weeks after the appearance of the first contractions, the contracting muscle fibers developed morphologic features strikingly different from those of muscle fibers contacted by neurites but not contracting. Mainly, the contracting fibers were considerably longer and thinner and were more densely packed in parallel to one another (Fig. $2, a, d, e)$. Measurements of the length and width of contracting and noncontracting muscle fibers were performed as follows: In 6 different experiments in which muscle was cocultured with the spinal cord explants for 28-57 d, overlapping photographs were taken in each culture dish of 8 contracting areas (in which individual muscle fibers could be well-visualized) and of 8 sister noncontracting arcas in the samc dish. After a montage (final magnification, $320 \times$ ) was made, the length of the muscle fibers was measured, and the width of the fibers was measured at 4 points per muscle fiber, which was arbitrarily divided into 5 equal parts. A total of 160 contracting muscle fibers and 174 muscle fibers contacted by neurites but not contracting was measured. The length and mean width of each muscle fiber were calculated. For the width, the following additional calculations were made, separately for the contracting and noncontracting muscle fibers: $(a)$ coefficient of variation (CV) $(\mathrm{SD} /$ mean $\times 100)$ for each individual muscle fiber; $(b)$ the mean of all individual fiber means in one experiment; $(c) \mathrm{CV}$ of all fibers in one experiment; and $(d)$ the mean of the CVs between the 8 experiments (calculation I). The means of " $\mathrm{d}$ " were compared between contracting and noncontracting fibers. Also calculated for the width was the "supermean" of the individual fiber CVs, taking all 8 cxpcriments together (calculation II), and comparison was made between contracting and noncontracting fibers.

\section{Results}

\section{Observation of living cultures}

The beginning of coculture, or "innervation," was arbitrarily considered to be the day the neural explants were added. Two to three days after coculture, long neurites had emerged from the spinal cord explants, and bouton-like structures were present at the nerve-muscle contacts (Fig. $1, a, b$ ). Neurites emerged earlier from the explants of WSC + DRG (24 hr of coculture) than from VSC or WSC explants (46-64 hr of coculture). However, after 5-6 d of coculture, the outgrowth of neurites from all 3 kinds of spinal cord preparation appeared to be the same.

In WSC + DRG cocultures, occasional asynchronous contractions of a few individual muscle fibers were observed as early as 4-6 d after coculture. Usually, though, synchronous contractions of several muscle fibers were not observed sooner than 10-12 d after coculture.

About 14-16 d after coculture, the number of contracting muscle fibers progressively increased, and the majority of contracting fibers had become entirely cross-striated (Fig. 1c). About 

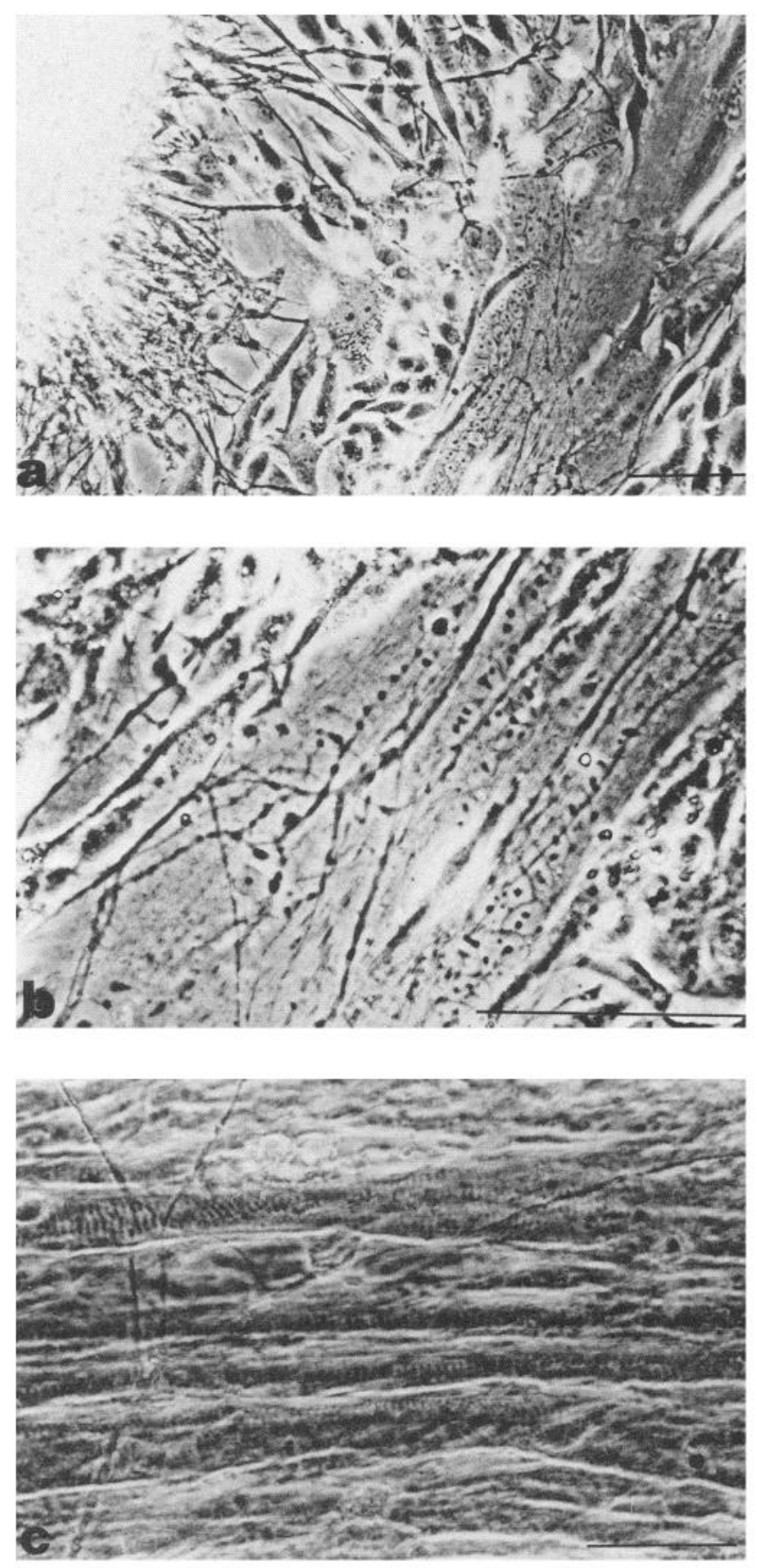

Figure 1. Phase-contrast microscopy of living muscle cultures. $a$, Lowpower photomicrograph of human muscle cocultured with an explant of WSC + DRG for $48 \mathrm{hr}$. There is an extensive outgrowth of neurites from the spinal cord explant. Bar, $100 \mu \mathrm{m}$. $b$, Higher-power photomicrographs of $a$. Abundant neurites are making contacts with the muscle fibers, and cause a bouton-like configuration to appear. Bar, $100 \mu \mathrm{m} . c$, Three weeks after coculture, muscle fibers contacted by neurites are entirely cross-striated. Bar, $50 \mu \mathrm{m}$.

16-20 d after coculture, the large areas of muscle fibers contracting in a continuous rhythm became morphologically distinct from the noncontracting areas, and they were maintained in that condition for up to 4-5 months. Densely packed contracting muscle fibers were present predominantly in close prox- imity to the ventral parts of the spinal cord explants (Fig. 2a). In long-term culture (after 3-4 weeks of coculture), muscle fibers around the dorsal parts of the spinal cord explants progressively degenerated (Fig. $2 a$ ). This was the case in $93 \%$ of the contracting areas. DRG neurons were well maintained in the long-term WSC + DRG cocultures (Fig. 2b). After several weeks of coculture, myelinated nerve fibers making contact with the muscle fibers were observed (Fig. 2c).

Contracting muscle fibers were thinner and much longer than noncontracting muscle fibers (Fig. $2, d, e$ ). The lengths of contracting and noncontracting muscle fibers were $19.0 \times 10^{2} \pm$ $18.0 \mu \mathrm{m}( \pm \mathrm{SEM})$ and $5.3 \times 10^{2} \pm 7.9 \mu \mathrm{m}$, respectively, based on measurement of 160 contracting and 174 noncontracting muscle fibers $(p<0.005)$.

Widths of contracting and of noncontracting muscle fibers were $8.9 \pm 0.07 \mu \mathrm{m}( \pm \mathrm{SEM})$ and $21.4 \pm 0.41 \mu \mathrm{m}$, respectively $(p<0.005)$.

In the 8 experiments, the mean $\mathrm{CV}$ of the width of contracting versus noncontracting muscle fibers (calculation I; see Materials and Methods) was $28.1 \pm 1.4 \mu \mathrm{m}$ (SEM) and $34.6 \pm 2.5 \mu \mathrm{m}$ $(p<0.025)$. The super-mean of the CV of the width of individual contracting and of noncontracting muscle fibers (calculation II; see Materials and Methods) was $23.0 \pm 1.6 \mu \mathrm{m}$ and $36.2 \pm 0.4 \mu \mathrm{m}$, respectively $(p<0.005)$. The 2 measurements indicate (1) that there was much less variation among different contracting muscle fibers in width, and (2) that the width of the individual contracting muscle fibers was considerably more constant along the fiber than it was along individual noncontracting muscle fibers.

\section{Quantitative analysis of AChR clusters on human muscle fibers cocultured with different types of rat spinal cord preparation}

Muscle fibers that were contacted by neurites growing from each kind of spinal cord explant had AChR clusters at the nervemuscle contacts (Fig. 3, $a, b$ ). By contrast, muscle fibers in the same culture dish that were not contacted by neurites were virtually free of AChR clusters (Fig. 3c). Five d after coculture, the number of $\mathrm{AChR}$ clusters per muscle fiber was significantly higher in WSC + DRG cocultures than in the other 2 sets. This difference gradually decreased after 12 and $21 \mathrm{~d}$ of coculture (Fig. 4a). There was also a slight but significant increase of AChR clusters in cocultures with WSC + DRG and WSC between 5 and $12 \mathrm{~d}$ of coculture (Fig. 4a). Muscle fibers cocultured with VSC had the lowest number of AChR clusters per muscle fiber at all 3 time points examined (Fig. $4 a$ ).

Twenty-one $d$ after coculture of muscle with WSC + DRG, there were large areas of contracting muscle fibers. Those fibers had a significantly $(p<0.005)$ lower number of AChR clusters per fiber $(1.7 \pm 0.15)$ compared to muscle fibers contacted by neurites but noncontracting in the same coculture $(3.21 \pm 0.21)$ (Figs. $4 a ; 3, d, e$ ). The percentage of muscle fibers contacted by neurites that contained AChR clusters was moderately higher in WSC + DRG cocultures at all 3 time points examined (Fig. $4 b)$. In the WSC + DRG coculture system, that percentage significantly increased $(p<0.025)$ from 5 to $12 \mathrm{~d}$ of coculture (Fig. $4 b$ ). In the other 2 types of coculture, the increase from 5 to $12 \mathrm{~d}$ was not significant. The percentage of contracting muscle fibers containing AChR clusters was much higher than in neurite-contacted but noncontracting muscle fibers within the same spinal cord coculture dishes (Fig. $4 b$ ). 

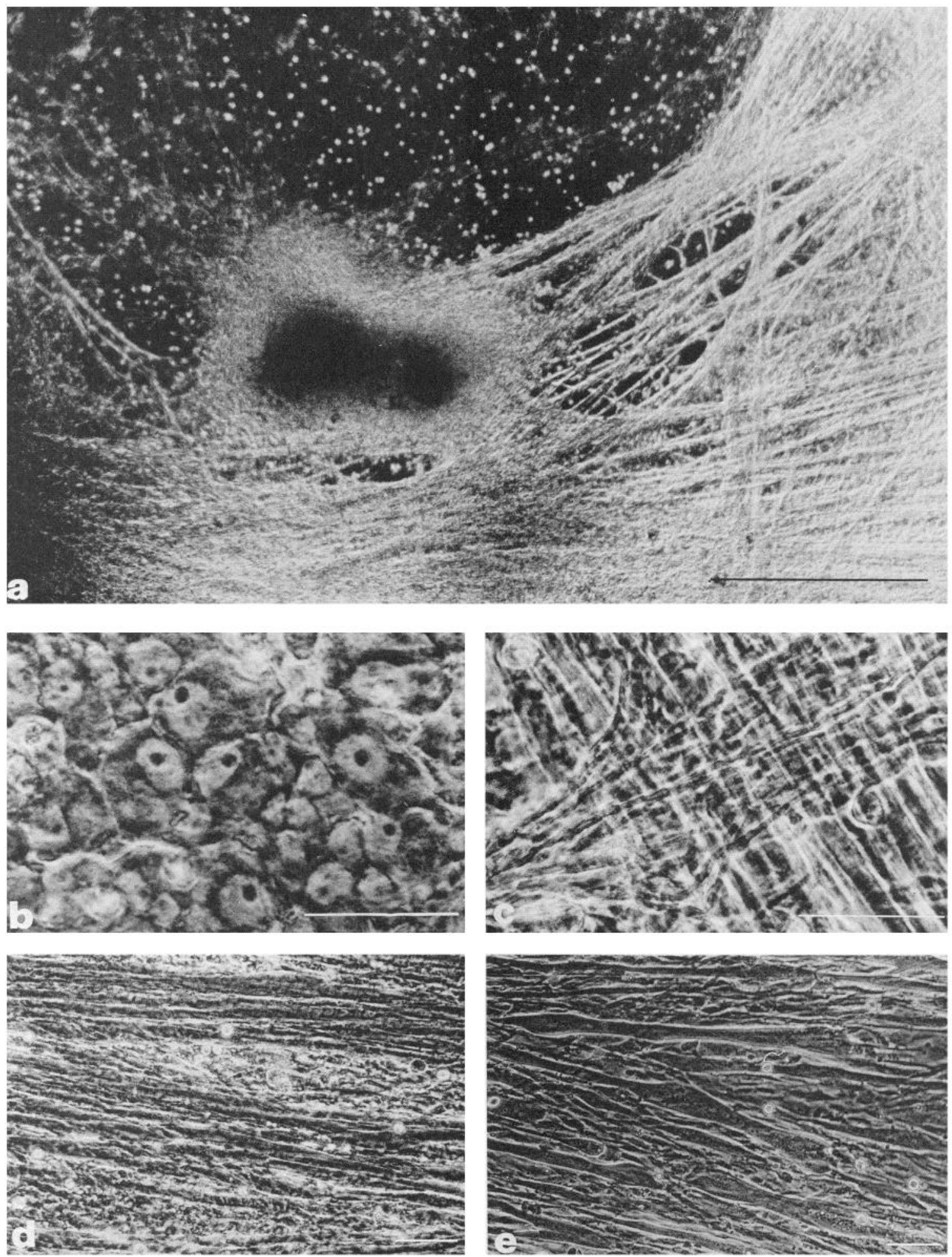

Figure 2. Phase-contrast microscopy of long-term cocultures of human muscle and WSC + DRG. $a$, Low-power photomicrograph of 9-week-old coculture. Abundant muscle fibers in close proximity to the presumably ventral part of the spinal cord explant are densely packed, parallel to each other, and are continuously contracting. Muscle fibers in proximity to the other (presumably dorsal) part of the spinal cord explant have degenerated. Bar, $1 \mathrm{~mm} . b$, Well-maintained DRG neurons in 4-week-old coculture. Bar, $50 \mu \mathrm{m}$. $c$, Myelinated nerve fibers branching over muscle fibers (from left to right) in 5-week-old coculture. Bar, $50 \mu \mathrm{m} . d, e$, Contracting $(d)$ and noncontracting $(e)$ muscle fibers in 3-week-old coculture. Contracting muscle fibers are thinner, longer, and more uniform in their individual diameters than noncontracting sister muscle fibers. Bar, $100 \mu \mathrm{m}$. 

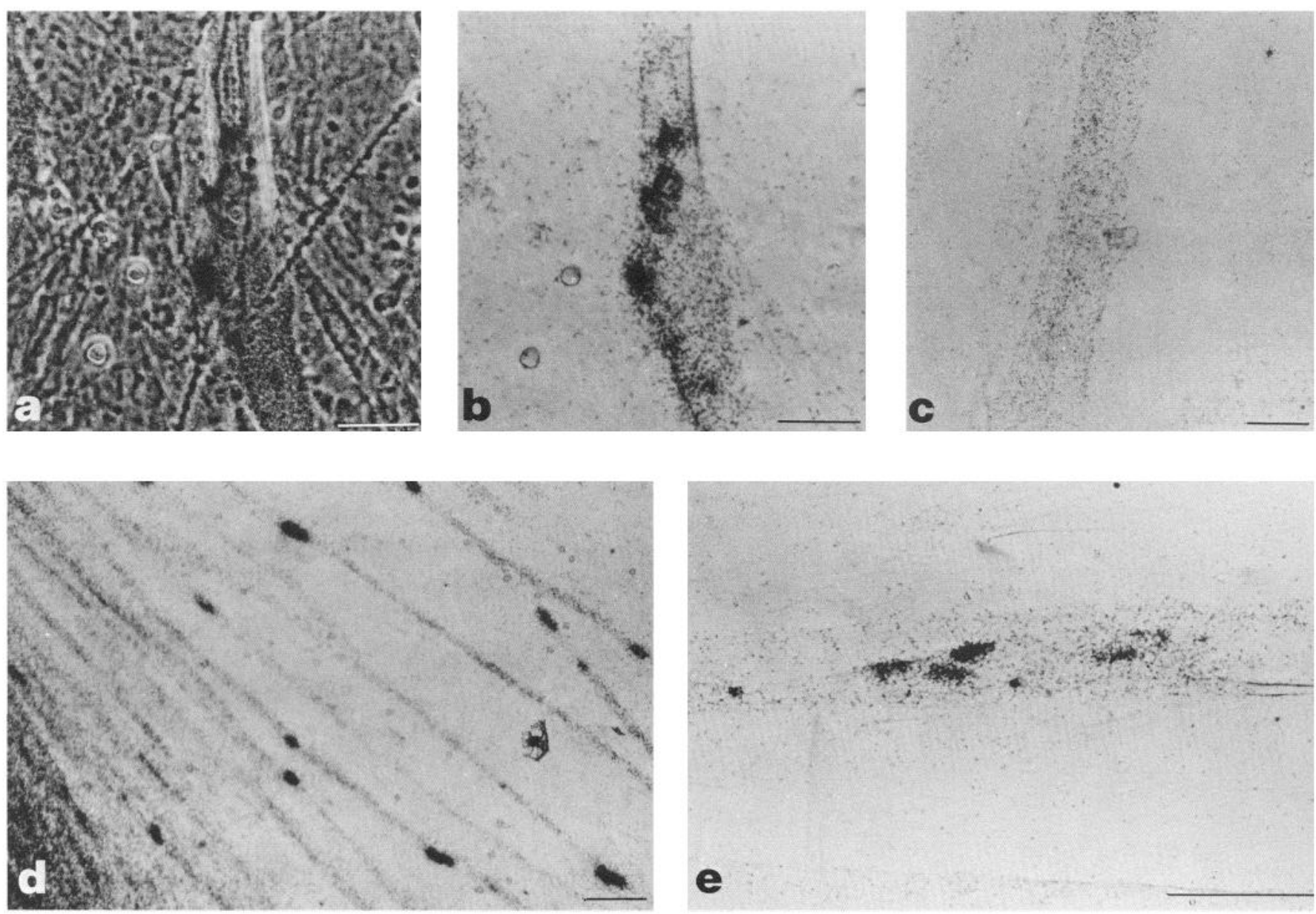

Figure 3. Autoradiography of ${ }^{125} \mathrm{I}$-alpha-BT. $a$, Phase-contrast and $(b)$ bright-field microscopy of a muscle fiber contacted by neurites but noncontracting, 3 weeks after coculture with WSC + DRG. Numerous AChR clusters are at the nerve-muscle contacts. Bar, $50 \mu \mathrm{m}$. $c$, Bright-field microscopy of a muscle fiber in the same dish as $a$, but far distant from the spinal cord explant. AChR clusters are lacking. Bar, $50 \mu$ m. $d$, Lowpower photomicrograph of innervated contracting muscle fibers after 3 weeks of coculture with WSC + DRG. Only 1 or 2 AChR clusters per muscle fiber are present. Bar, $100 \mu \mathrm{m}$. $e$, A muscle fiber contacted by neurites but noncontracting has several AChR clusters after 9 weeks of coculture with WSC + DRG. Bar, $100 \mu \mathrm{m}$.

\section{Quantitative analysis of AChE patches}

AChE-positive patches were quantatively analyzed at the same 3 time points of coculture as were $\mathrm{AChR}$ clusters, but in different experiments. In addition, $\mathrm{AChE}$ patches on contracting and noncontracting muscle fibers in the same dish were examined after 31-64 d of coculture. By $5 \mathrm{~d}$ of coculture, AChE-positive patches had appeared on muscle fibers contacted by neurites. The number of AChE patches per muscle fiber was practically the same in all 3 kinds of spinal cord coculture and remained constant at the 5, 12, and $21 \mathrm{~d}$ time points (Fig. 5a). However, after 31-64 d of coculture, the contracting muscle fibers had a significantly lower $(p<0.005)$ number of AChE-positive sites (mean, $1.5 \pm 0.17$ per muscle fiber) than did muscle fibers contacted by neurites but noncontracting in the same culture dish (mean, $2.5 \pm 0.12$ per muscle fiber) (Fig. $5 a$ ).

Among all the fibers contacted by neurites, the percentage of muscle fibers containing AChE patches remained the same at the 3 time points examined in VSC and WSC cocultures, but significantly increased $(p<0.005)$ between 12 and $21 \mathrm{~d}$ of coculture with WSC + DRG (Fig. $5 b$ ). The percentage of contracting muscle fibers containing AChE-positive sites was sig- nificantly higher $(p<0.005)$ than of noncontracting muscle fibers containing AChE-positive sites in the same dish (50.3 \pm 6.9 and $25.8 \pm 0.6 \%$, respectively) (Fig. $5 b$ ).

In muscle fibers contacted by neurites but noncontracting, small linear patches of AChE staining were present at the nervemuscle contact (Fig. 6a). Well-organized, complex AChE patches resembling those of adult human muscle fibers were present only on contracting muscle fibers (Fig. $6 b$ ). Muscle fibers not contacted by neurites had virtually no AChE stain.

Five to ten weeks after coculture, organized $\mathrm{AChE}$ structures constituted $36.6 \pm 6.5 \%$ of the total number of $\mathrm{AChE}$ patches on contracting muscle fibers.

\section{Double-labeling of $A C h R$ and $A C h E$}

On noncontracting muscle fibers, only $44 \%$ of the AChR clusters had identifiable AChE patches, but $93.4 \%$ of $A C h E$ patches had corresponding AChR clusters (Fig. 7, $a, b$ ). By contrast, on contracting muscle fibers, $64 \%$ of AChR clusters had corresponding AChE-positive sites, and $98 \%$ of AChE sites had corresponding $\mathrm{AChR}$ clusters.

On individual muscle fibers, the average length of a simple 
$\mathbf{a}$
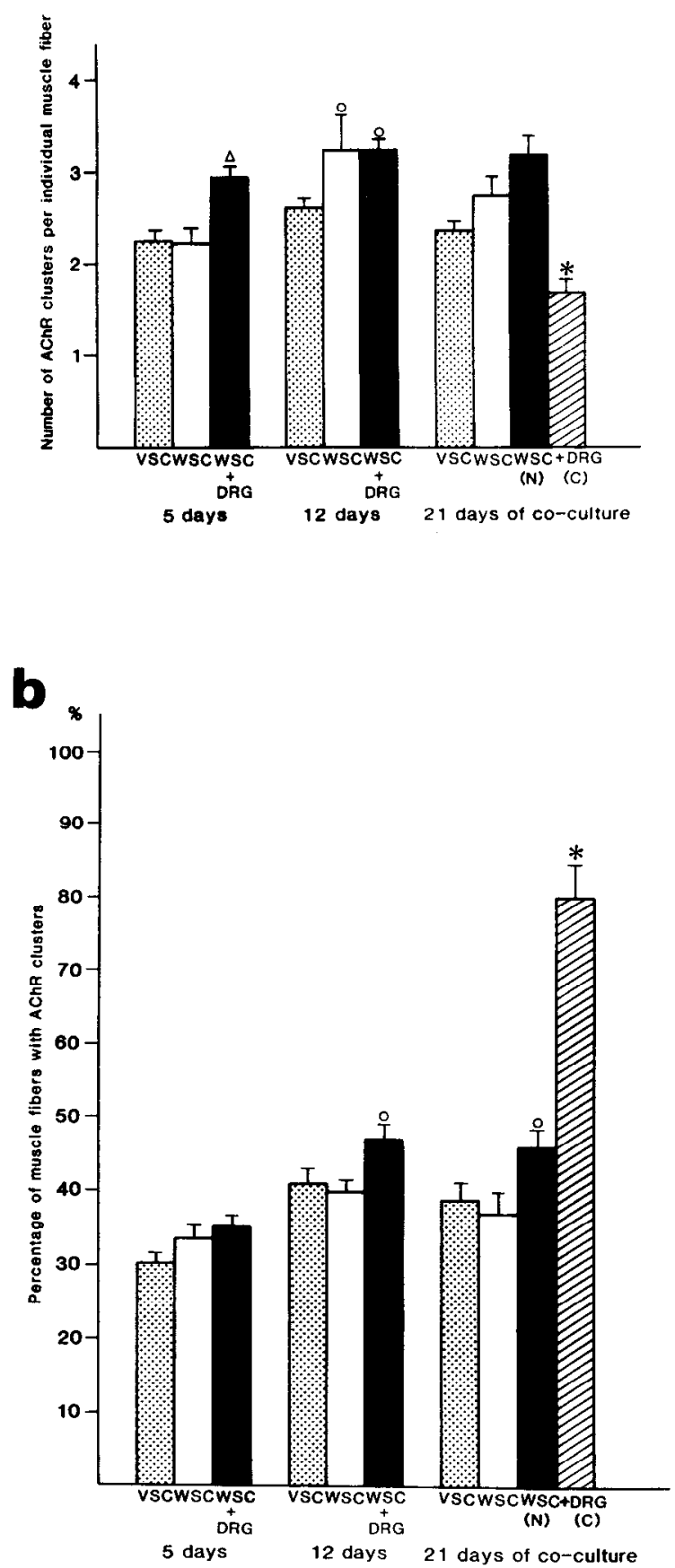

Figure 4. AChR clusters in muscle cocultured with three kinds of spinal cord explant (details in text): VSC, ventral part of spinal cord; $W S C$, whole spinal cord; $W S C+D R G$, whole spinal cord with dorsal root ganglia attached $-(N)$, noncontracting or $(C)$ contracting muscle fibers. $a$, Number of AChR clusters per muscle fiber. After $5 \mathrm{~d}$ of coculture, there were significantly more AChR clusters in the WSC + DRG cocultures $(\triangle, p<0.01)$ than in VSC and WSC cocultures. After $12 \mathrm{~d}$ of coculture, there was a moderate but statistically significant increase, as compared to after $5 \mathrm{~d}$ of coculture, in the number of $\mathrm{AChR}$ clusters in WSC + DRG and WSC coculturcs $(O, p<0.05)$. After 3 weeks of coculture, the number of AChR clusters was dramatically reduced in contracting $(C)$ muscle fibers in WSC + DRG cocultures compared to in noncontracting $(N)$ sister muscle fibers in the same culture dishes $\left(^{*}, p<0.005\right)$. $b$, Percentage of muscle fibers containing AChR clusters. After 12 and $21 \mathrm{~d}$ of coculture, there were significantly more muscle fibers with AChR clusters in WSC + DRG cocultures than after $5 \mathrm{~d}$ of coculture with WSC + DRG $(*, p<0.025)$. After $21 \mathrm{~d}$ of a

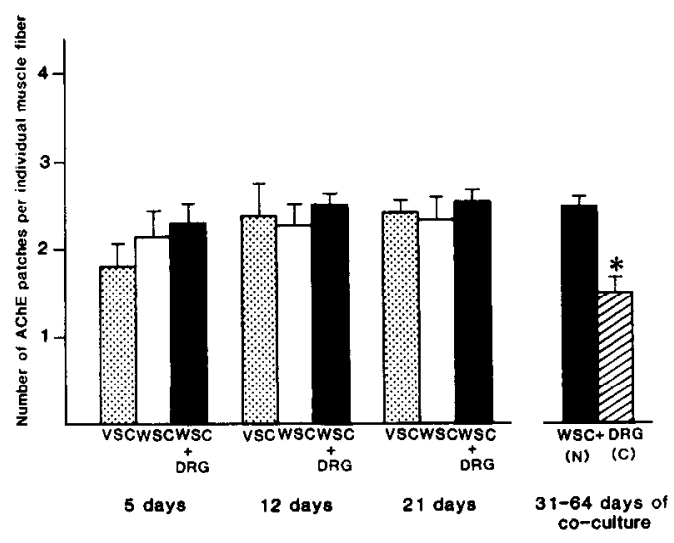

b

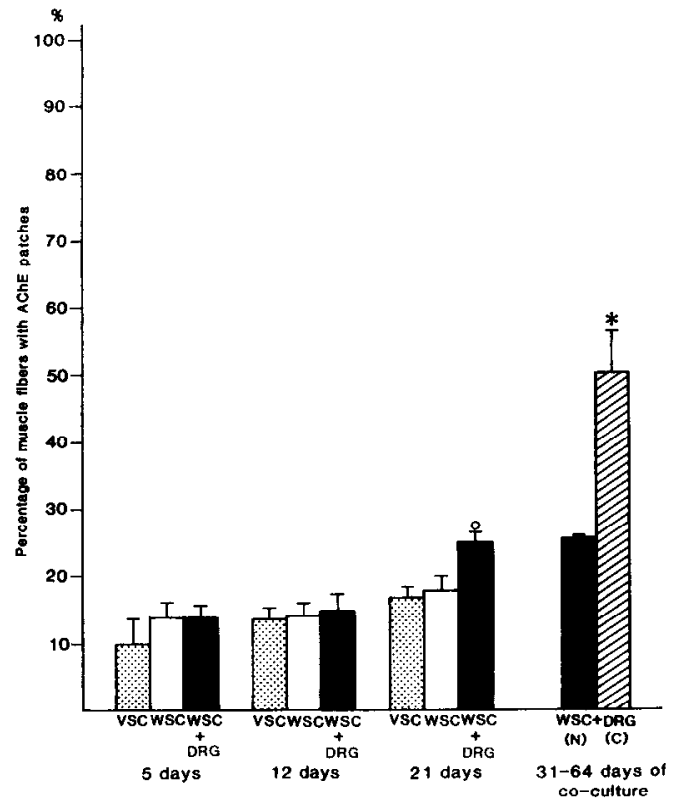

Figure 5. AChE patches in muscle cocultured with 3 kinds of spinal cord explants. Symbols are as in legend of Figure 4. $a$, Number of AChE patches per muscle fiber. The number of $\mathrm{AChE}$ patches per muscle fiber remained virtually constant from 5 to $21 \mathrm{~d}$ of coculture with all 3 kinds of spinal cord explants. From 31 to $64 \mathrm{~d}$ of coculture with WSC + DRG, contracting $(C)$ muscle fibers had a significantly decreased number of AChE patches $\left(^{*}, p<0.005\right)$. $b$, Percentage of muscle fibers containing AChE patches. There was a significant increase in the number of fibers containing AChE patches at $21 \mathrm{~d}$ of culture as compared to after 5 and $12 \mathrm{~d}$ of coculture with WSC $+\operatorname{DRG}(\mathrm{O}, p<0.025)$. From 31 to $64 \mathrm{~d}$ of coculture with WSC + DRG, the percentage of contracting $(C)$ muscle fibers containing $\mathrm{AChE}$ patches was much larger than that of noncontracting $(N)$ sister muscle fibers in the same culture dishes $\left({ }^{*}, p<0.005\right)$.

coculture, the percentage of contracting $(C)$ muscle fibers having $\mathrm{AChR}$ clusters was much larger than that of noncontracting $(N)$ sister fibers in the same culture dishes $\left(^{*}, p<0.005\right)$. 

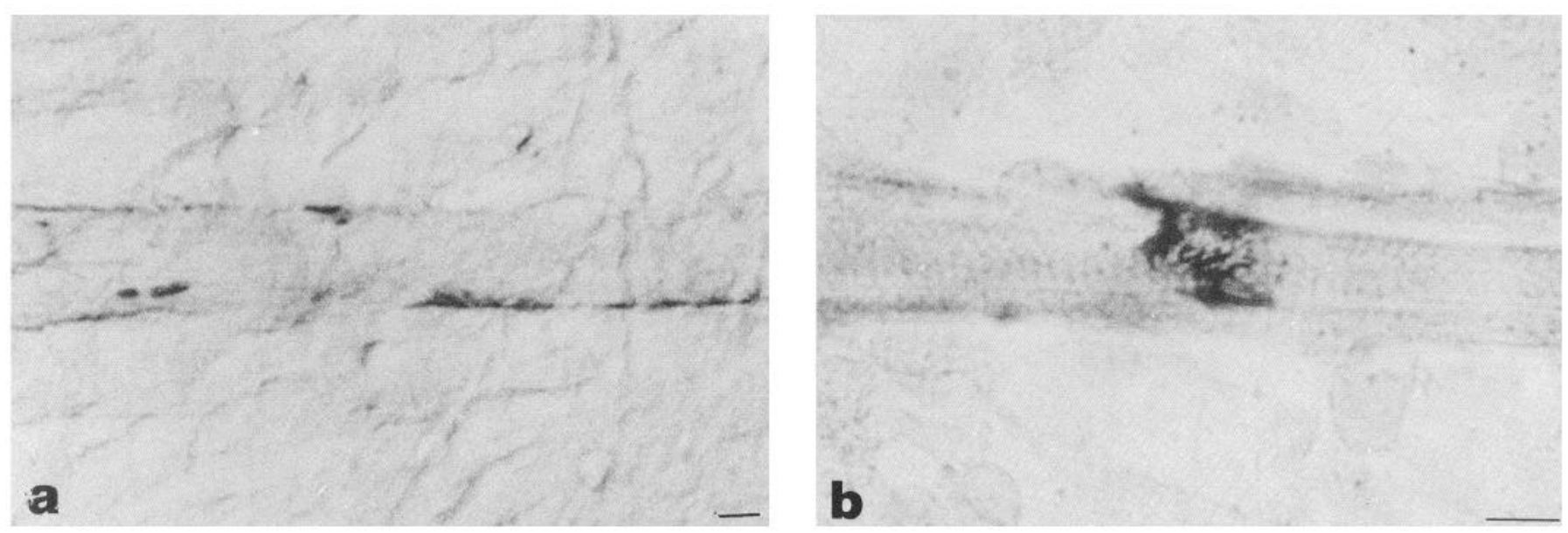

Figure 6. Appearance of AChE patches on muscle fibers after 5 weeks of coculture with WSC + DRG. $a$, Muscle fiber contacted by neurites but noncontracting has several linear AChE patches. Bar, $10 \mu \mathrm{m}$. $b$, Contracting muscle fiber has one well-organized AChE patch. Bar, $10 \mu \mathrm{m}$.

AChE-positive site was $57 \%$ smaller $(p<0.005)$ than the length of an AChR cluster (Fig. 7, $a, b$ ). The average length of an unorganized AChE patch on a contracting muscle fiber was $65 \%$ smaller $(p<0.005)$ than the length of an AChR cluster (Fig. 8, $a, b)$. However, the average length of an organized AChE patch on a contracting muscle fiber was virtually the same as the length of an AChR cluster (Fig. 8, c, d). The average length of an AChR cluster without corresponding AChE-positive patches was smaller than an AChR cluster with a corresponding AChE patch on both noncontracting and contracting muscle fibers -30 and $16 \%$, respectively.

\section{Discussion}

These studies demonstrate the importance of dorsal root ganglia in our spinal cord and human muscle coculture system for achieving successful innervation of the cultured human muscle by rat motor neurons. We consider the innervation successful when the following features are present: (1) contractions of the muscle fibers, which can be inhibited by $d$-tubocurarine; (2) entirely cross-striated muscle fibers; (3) well-organized AChEpositive sites; and (4) unifocal innervation, having changed from multifocal innervation. Since successful innervation was achieved in coculture of human muscle only with WSC + DRG and not in cocultures with VSC or WSC, the question arose as to whether this difference was paralleled by developmental differences in AChR clustering and AChE accumulation at the nerve-muscle contacts in those various coculture systems. (Human muscle cocultured with DRG alone did not contract and did not have AChR clusters and AChE-positive sites; V. Askanas and T. Kobayashi, unpublished observations).

To our knowledge, this report provides the first systematic description of the development of AChR clusters and AChEpositive sites at the nerve-muscle contacts in animal or human muscle cocultured with different types of spinal cord explants. Human muscle cultured in monolayer is very suitable for this kind of study because, when cultured aneurally, it does not spontaneously contract, does not have AChR clusters, and does not have identifiable AChE staining (Askanas et al., 1977; Kobayashi and Askanas, 1985). In addition, when cultured in monolayer and innervated by fetal rat spinal cord, the human muscle survives as long as 4-5 months, allowing precise analysis of the events associated with early, mid-term, and long-term innervation. Since not all muscle fibers contacted by neurites contracted, the difference between noncontracting and contracting muscle fibers could be analyzed.

Previous pioneering work by Crain et al. (1970) has established that cultured human muscle can, in what they term an "organotypic" muscle preparation, be innervated by. neurons from fetal mouse spinal cord with DRG attached. Explants in their organotypic system consisted of a bundle of teased rodent or human muscle fibers. Within the explant, regenerating muscle fibers were said to replace the original ones and become innervated (Crain et al., 1970; Peterson and Crain, 1972, 1979). The same authors subsequently reported that innervation of adult mouse muscle in organotypic culture can be obtained with the ventral part of fetal mouse spinal cord only, although that innervation was said to be considerably reduced as compared to innervation achieved by the spinal cord with DRG attached. However, a detailed comparison, such as we present of the effects of different types of spinal cord coculture, has not been reported regarding innervation of human muscle fibers, nor has the role of whole spinal cord with and without DRG. In the organotypic culture model, new muscle fibers form within the original explant, and apparently within the basal lamina of the originally explanted muscle fibers (Peterson and Crain, 1972, 1979; Ecob, 1984). That organotypic system is very different from our system of monolayer culture of human muscle. In our system, new muscle fibers in monolayer cultures developed entirely from outgrowing myoblasts, without any remnants of original basal lamina, because the original explants were removed after the myoblasts started emerging from them. It is well-established that, during muscle regeneration in vivo, neuromuscular synapses typically form at the original synaptic sites, apparently marked by chemical specialization at those sites on the persisting original basal lamina (Marshall et al., 1977; Sanes et al., 1978; Burden et al., 1979). In organotypic mouse musclefetal mouse spinal cord cocultures, Ecob (1984) has postulated that neuromuscular junctions may form at both ectopic and the original synaptic sites; this point has not yet been studied in organotypic human muscle cocultures. For these reasons, we favor the monolayer culture of human muscle as a model that more easily permits complete evaluation of the events associ- 



Figure 7. Double-labeling of AChRs and AChE patches on muscle fibers contacted by neurites but noncontracting in WSC + DRG 3 week cocultures. $a$, Histochemistry of AChE. $b$, Autoradiography of ${ }^{125} \mathrm{I}$-alpha-BT. AChR clusters in $b$ are more numerous and larger than the AChE patches; an AChR cluster and an AChE patch are present together in only one area (arrow). Bar, $50 \mu \mathrm{m} . c$, Histochemistry of AChE. $d$, Autoradiography of ${ }^{125} \mathrm{I}$-alpha-BT. In this area all AChR clusters have corresponding AChE patches. Bar, $10 \mu \mathrm{m}$.

ated with de novo formation of neuromuscular junctions.

In our studies, as compared to VSC or WSC cocultures, explants of WSC + DRG-induced more AChR clusters per individual innervated muscle fiber after $5 \mathrm{~d}$ of coculture, and the percentage of muscle fibers containing one or more AChR clusters was moderately higher in WSC + DRG cocultures at all 3 time points quantitated.

While the great majority (93.4\%) of AChE patches on noncontracting muscle fibers and virtually all (98\%) of $\mathrm{AChE}$ patches on contracting muscle fibers had corresponding AChR clusters, a much lower percentage ( $44 \%$ on noncontracting and $64 \%$ on contracting muscle fibers) of AChR clusters had corresponding $\mathrm{AChE}$ patches on the same muscle fibers. This, and the fact that there were significantly more $\mathrm{AChR}$ clusters than $\mathrm{AChE}$ patches on noncontracting muscle fibers, indicates that detectable $\mathrm{AChR}$ clusters are more easily induced at the nerve-muscle contacts than are $\mathrm{AChE}$ accumulations. Interestingly, while the number of muscle fibers containing AChR clusters was significantly higher in WSC + DRG coculture at all 3 time points examined, the number of muscle fibers containing AChE-positive sites increased significantly only between 12 and $21 \mathrm{~d}$ of coculture with WSC + DRG. This finding, and the fact that there was also a significant increase in the number of contracting muscle fibers between 12 and $21 \mathrm{~d}$ of coculture with WSC + DRG, suggests that in this period of time there may be an increase in acetylcholine release inducing both the muscle contractions and the AChE-positive sites.

The mechanism(s) by which DRG contribute to motor neu- ron-induced contractions in our culture system is not clear. From the literature it is known that DRG have a trophic influence on spinal motor neurons in vivo. Early removal of DRG during embryogenesis of tadpoles caused reduction of surviving motor neurons (Davis et al., 1983). In the chick embryo, removal of DRG afferent fibers resulted in increased loss of motor neurons during the final stages of natural motor neuron death (Okado and Oppenheim, 1984). Efferent terminals of DRG neurons form synapses with both motor neuron dendrites and motor neuron somas in the spinal cord of frog and cat (McLaughlin, 1972; Liuzzi et al., 1984). In the developing tadpole larva, DRG afferents contact migrating motor neurons (Liuzzi et al., 1983). Recently it has been reported that chick motor neurons in homogeneous cultures can be kept viable for a longer period of time if DRG neurites make physical contact with them (Bennett, 1986). From these reports, it can be concluded that DRG exert a trophic (survival?) influence on motor neurons. Exactly how DRG exert their seemingly necessary trophic or/and excitatory influence on motor neurons in our culture system (and in the other systems) remains to be studied.

In monolayer cultures of embryonic chick muscle, functional nerve-muscle contacts were formed de novo in cocultures with spinal cord without DRG, and spontaneous and stimulus-evoked action potentials were detected in myotubes adjacent to the spinal cord explants within $24 \mathrm{hr}$ after coculture with the spinal cord explants (Fischbach, 1972; Frank and Fischbach, 1977). Since cultured chick embryo muscle fibers spontaneously contract even in aneural cultures, the influence of innervation on 

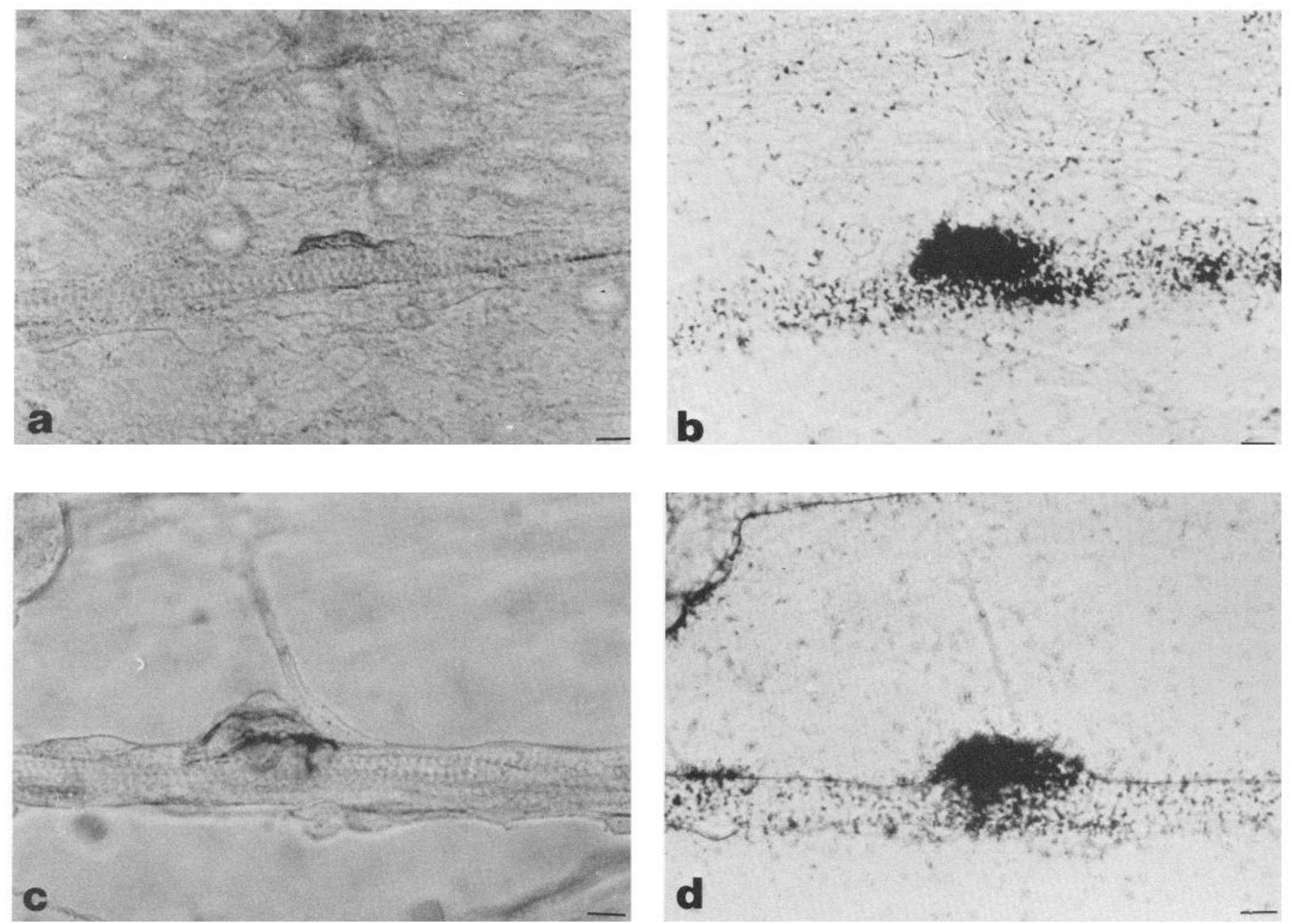

Figure 8. Double-labeling of AChE and AChR on muscle fibers innervated and contracting in WSC + DRG 5 week coculture. $a$, AChE. $b$, Autoradiography of ${ }^{125} \mathrm{I}$-alpha-BT of a simple unorganized nerve-muscle contact. Note that the AChR cluster is much larger than the AChE patch. Bar, $10 \mu \mathrm{m}$. $c$, AChE. $d$, Autoradiography of ${ }^{125} \mathrm{I}$-alpha-BT of a well-organized neuromuscular junction. The size of the AChE patch is virtually the same as that of the AChR cluster. Bar, $10 \mu \mathrm{m}$.

the induction of muscle contractions in that system is not as critical as it is with cultured human muscle fibers.

In our culture system, contracting human muscle fibers innervated by WSC + DRG expressed several properties of developing innervated muscle in vivo. Namely, the population of contracting muscle fibers was considerably longer, showed a trend toward uni-endplate status, and had a complicated AChEpositive site at the nerve-muscle contact.

Loss of multi-endplate status of muscle fibers during development in vivo is a well-recognized phenomenon (Redfern, 1970; Brown et al., 1976; Dennis et al., 1981; Bennett, 1983). It has been suggested that, while clustering of AChRs at the nervemuscle contact does not depend on muscle activity, the loss of multi-endplate status and accumulation of AChE may depend on muscle activity (Giacobini et al., 1973; Lømo and Slater, 1978; Rubin et al., 1980; and review in Bennett, 1983). Development of organized neuromuscular junctions in the rat has also been considered to be linked to muscle activity (Lømo et al., 1984).

Our double-labeling studies of AChRs plus AChE showed that, in noncontracting immature muscle fibers, $\mathrm{AChE}$ patches are linear and shorter in length than AChR clusters. However, contracting muscle fibers exhibited greater organization of the nerve-muscle contact, expressed by a large and complicated AChE-positive site. Whether, in our culture system, the transition from multifocal to unifocal innervation and the enhanced organization of the neuromuscular junction might have been at least partly influenced by muscle fiber activity is not known. Our recently initiated experiments studying a variety of parameters in contracting, innervated cultured human muscle fibers versus innervated but paralyzed (by tetrodotoxin or alpha-BT) cultured muscle should help distinguish which parameters of innervation depend on muscle activity and which are strictly neurally regulated.

Another point derived from our studies relates to muscle fiber growth. In our culture system, similar to the situation in vivo, innervated and contracting muscle fibers became considerably longer than muscle fibers contacted by neurites but not contracting. Even though the mean width of the individual contracting innervated muscle fibers was smaller than that of muscle fibers contacted by neurites but not contracting, there was a definite increase of a total mass of muscle (more muscle fibers) in the innervated regions. Growth of muscle fibers in vivo has been linked to both innervation and activity (Williams and 
Goldspink, 1971). Recently, the nerve-muscle contact and the subsequent activity have been emphasized as the important steps in vivo in the formation of secondary myotubes, from which a majority of the adult muscle fibers generate (Harris, 1986). Whether the increase of muscle mass in our culture system reflects the development of secondary myotubes or is due to other phenomena remains unknown.

\section{Conclusion and prospects}

The developmental pattern of innervation of human muscle cocultured in monolayer with fetal rat spinal cord having DRG attached resembles that of innervated muscle in vivo. Our culture system provides a useful experimental model, in which short-, mid-, and long-term events associated with de novo innervation of human muscle can be precisely studied. Since human fetal material is practically not available for this kind of systematic study, tissue culture of human muscle is becoming an important model for multidisciplinary analyses of human muscle development and innervation. Recent studies from our laboratory have demonstrated that expression of genes for the musclespecific isozymes of creatine kinase, glycogen phosphorylase, and phosphoglyccratc mutasc was significantly increased in human muscle cultured in monolayer and innervated by WSC + DRG (Martinuzzi et al., 1986, 1987). Histoenzymatic maturation of innervated cultured human muscle was also much more advanced than that of the noninnervated controls (Vita et al., 1987). In addition, the innervated cultured human muscle fibers had increased resting membrane potentials compared to the noninnervated cultured human fibers, and they exhibited curare-sensitive miniature endplate and endplate potentials, as demonstrated by microelectrode studies (Saito et al., 1986; K. Saito, T. Kobayashi, V. Askanas, W. K. Engel, and K. Ishikawa, unpublished observations).

Aneural monolayer cultures of diseased adult human muscle have already provided important information regarding pathogeneses of some neuromuscular disorders (Askanas, 1984; Witkowski, 1986a, b). Our new model of de novo innervation of human muscle cultured in monolayer can now be applied to study diseased human muscle, especially those diseases that may require innervation for phenotypic manifestation of muscle-cell abnormalities.

\section{References}

Askanas, V. (1984) Human muscle and Schwann cells in tissue culture as a tool in studying pathogenesis and treatment of neuromuscular disorders. In Neuromuscular Diseases, G. Serratrice et al., eds., pp. 373-379, Raven, New York.

Askanas, V., and W. K. Engel (1975) New program for investigating adult human skeletal muscle grown aneurally in tissue culture. Neurology $25: 58-67$

Askanas, V., and W. K. Engel (1979) Normal and diseased human muscle in tissue culture. In Handbook of Clinical Neurology, Vol. 40 , P. J. Vinken and G. W. Bryun, eds., pp. 183-196, North-Holland, New York.

Askanas, V., and G. Gallez-Hawkins (1985) Synergistic influence of polypeptide growth factors on cultured human muscle. Arch. Neurol. 18: $716-719$

Askanas, V., W. K. Engel, S. Ringel, and A. Bender (1977) Acetylcholine receptors in aneurally cultured human and animal muscle. Neurology 27: 1019-1022.

Askanas, V., W. K. Engel, and T. Kobayashi (1985) TRH enhances motor-neuron-evoked contractions of cultured human muscle. Ann. Neurol. 18: 716-719.

Bennett, M. R. (1983) Development of neurnmuscular syapses. Physiol. Rev. 63: 915-1048.
Bennett, M. R. (1986) Lower motoneurone afferent trophic factors and LMN death: Effect and mechanism. Muscle Nerve (Suppl.) 9: 15.

Bennett, M. R., and A. G. Pettigrew (1974) The formation of synapses in striated muscle during development. J. Physiol. (Lond.) 241:515545.

Brown, M. C., J. K. S. Jansen, and D. V. Essen (1976) Polyneural inncrvation of skcletal muscle in ncw-born rats and its climination during maturation. J. Physiol. (Lond.) 261: 387-422.

Burden, S. J., P. B. Sargent, and U. J. McMahan (1979) Acetylcholine receptors in regenerating muscle accumulate at original synaptic sites in the absence of the nerve. J. Cell Biol. 182: 412-425.

Crain, S. M., L. Alfei, and E. R. Peterson (1970) Neuromuscular transmission in cultures of adult human and rodent skeletal muscle after innervation in vitro by fetal rodent spinal cord. J. Neurobiol. 1 : 471-489.

Davey, D. F., and M. W. Cohen (1986) Localization of acetylcholine receptors and cholinesterase on nerve-contacted and noncontacted muscle cells grown in the presence of agents that block action potentials. J. Neurosci. 6: 673-680.

Davis, M. R., M. Constantine-Paton, and D. Shorr (1983) Dorsal root ganglia removal in Rana pipiens produces fewer motoneurons. Brain Res. 265: 283-288.

Dennis, M. J., L. Ziskind-Conhaim, and A. J. Harris (1981) Development of neuromuscular junctions in rat embryo. Dev. Biol. 81 . 266-279.

Ecob, M. (1984) The location of neuromuscular junctions on regenerating adult mouse muscle in culture. J. Neurol. Sci. 64: 175-182.

Fischbach, G. D. (1972) Synapse formation between dissociated nerve and muscle cells in low density cell culture. Dev. Biol. 28: 407-429.

Fischbach, G. D., E. Frank, T. M. Jessel, L. L. Rubin, and S. M. Scheutze (1979) Accumulation of acetylcholine receptors and acetylcholinesterase at newly formed nerve-muscle synapses. Pharmacol. Rev 30: 411-428.

Frank, E., and G. D. Fischbach (1977) ACh receptors accumulate at newly formed nerve-muscle synapses in vitro. In Cell and Tissue Interactions, J. W. Lash and M. M. Burger, eds., pp. 285-292, Raven, New York.

Giacobini, G., G. Filogamo, M. Weber, P. Boquet, and J. P. Changeux (1973) Effects of a snake alpha-neurotoxin on the development of innervated skeletal muscles in chick embryo. Proc. Natl. Acad. Sci. USA 70: 1708-1712.

Harris, A. J. (1986) Transfer of synaptic terminals from primary to secondary myotubes. Muscle Nerve (Suppl.) 9: 44.

Karnovsky, M. J., and L. Roots (1964) Direct-coloring of thiocholine method for cholinesterase. J. Histochem. Cytochem. 12: 219-221.

Kobayashi, T., and V. Askanas (1985) Acetycholine receptors and acetylcholinesterase accumulate at the nerve-muscle contacts of denovo grown human monolayer muscle co-cultured with fetal rat spinal cord. Exp. Neurol. 88: 327-335.

Kobayashi, T., V. Askanas, and W. K. Engel (1985) Innervation of human muscle cultured in monolayer by rat spinal cord is influenced by dorsal root ganglia (DRG). J. Cell Biol. (Suppl.) 101: 131 a.

Kobayashi, T., H. Tsukagoshi, and Y. Shimizu (1982) Trophic effects of sympathetic ganglia on normal and dystrophic chicken skeletal muscles in tissue culture. Exp. Neurol. 77: 241-253.

Liuzzi, F. J., M. S. Beattie, and J. C. Bresnahan (1983) Dorsal root afferents contact migrating motoneurons in the developing frog spinal cord. Brain Res. 262: 299-302.

Liuzzi, F. J., M. S. Beattie, and J. C. Bresnahan (1984) The relationship of dorsal root afferents to motoneuron somata and dendrites in the adult bullfrog: A light and electron microscopic study using horseradish peroxidase. Neuroscience 4: 951-961.

Lomo, T., and C. R. Slater (1978) Control of acetylcholine sensitivity and synaptic formation by muscle activity. J. Physiol. (Lond.) 275: $391-402$.

Lomo, T., R. Mirsky, and S. Pockett (1984) Formation of neuromuscular junctions in adult rats: Role of postsynaptic impulse activity. In Neuromuscular Diseases, G. Serratrice et al., eds., pp. 393399, Raven, New York.

Marshall, L. M., J. R. Sanes, and U. J. McMahan (1977) Reinnervation of original synaptic sites on muscle fiber basement membrane after disruption of the muscle cells. Proc. Natl. Acad. Sci. USA 74: 30733077.

Martinuzzi, A., V. Askanas, T. Kobayashi, W. K. Engel, and S. DiMauro (1986) Expression of muscle-gene specific isoenzymes of phosphor- 
ylase and creatine kinase in innervated cultured human muscle. J. Cell Biol. 103: 1423-1429.

Martinuzzi, A., V. Askanas, T. Kobayashi, W. K. Engel, and J. Gorsky (1987) Developmental expression of the muscle-specific isozyme of phosphoglycerate mutase in human muscle cultured in monolayer and innervated by fetal rat spinal cord. Exp. Neurol. 96: 365-372.

McLaughlin, B. J. (1972) Dorsal root projections to the motor nuclei in cat spinal cord. J. Comp. Neurol. 144: 461-474.

Nakajima, Y., Y. Kidokoro, and F. G. Klier (1980) The development of functional neuromuscular junctions in vitro: An ultrastructure and physiological study. Dev. Biol. 77: 52-72.

Nakamura, T., and K. Torigoe (1979) Simultaneous visualization of catecholamine fluorescence and cholinesierase activity in the peripheral autonomic nerve. Acta Histochem. Cytochem. 12: 569.

Okado, N., and R. W. Oppenheim (1984) Cell death of motoneurons in the chick embryo spinal cord. IV. In the loss of motoneurons following removal of afferent input. J. Neurosci. 4: 1639-1652.

Peterson, E. R., and S. M. Crain. (1972) Regeneration and innervation in cultures of adult mammalian skeletal muscle coupled with fetal rodent spinal cord. Exp. Neurol. 36: 136-159.

Peterson, E. R., and S. M. Crain (1979) Maturation of human muscle after innervation by fetal mouse spinal cord explants in long-term cultures. In Muscle Regeneration, A. Maruo, ed., pp. 429-441, Raven, New York.

Redfern, P. (1970) Neuromuscular transmission in new-born rat. J. Physiol. (Lond.) 209: 701-709.

Rubin, L., L. S. M. Schuetze, C. L. Weill, and G. D. Fischbach (1980)
Regulation of acetylcholinesterase appearance at neuromuscular junctions in vitro. Nature 238: 264-267.

Saito, K., T. Kobayashi, V. Askanas, W. K. Engel, and K. Ishikawa (1986) Electrical parameters of human muscle cultured in monolayer aneurally and innervated by rat spinal cord. Muscle Nerve (Suppl.) 9: 162.

Sanes, J. R., L. M. Marshall, and U. J. McMahan (1978) Reinnervation of muscle fiber basal lamina after removal of myofibers. Differentiation of regenerating axons at original synaptic sites. J. Cell Biol. 78 . 176-198.

Shimada, Y., D. A. Fishman, and A. A. Moscona (1969) Formation of neuromuscular junction in embryonic cell cultures. Proc. Natl. Acad. Sci. 62: 715-721.

Vita, G., V. Askanas, A. Martinuzzi, and W. K. Engel (1987) Histoenzymatic profile of human muscle cultured in monolayer and innervated de novo by fetal rat spinal cord. Muscle Nerve (in press).

Vogcl, Z., A. J. Sytkowsky, and M. W. Nierenberg (1972) Acetylcholine receptors of muscle grown in vitro. Proc. Natl. Acad. Sci. USA 69: 3180-3184.

Williams, P. E., and G. Goldspink (1971) Longitudinal growth of striated muscle fibers. J. Cell Sci. 9: 751-767.

Witkowski, J. A. (1986a) Tissue culture studies of muscle disorders: Part 1. Techniques, cell growth, morphology, cell surface. Muscle Nerve 9: 191-207.

Witkowski, J. A. (1986b) Tissue culture studies of muscle disorders: Part 2. Biochemical studies, nerve-muscle culture, metabolic myopathies and animal models. Muscle Nerve 9: 283-298. 